\title{
minarit
}

Revista Educación

ISSN: 0379-7082

ISSN: 2215-2644

revedu@gmail.com

Universidad de Costa Rica

Costa Rica

\section{Docentes de audición y lenguaje itinerante: el trabajo colaborativo, reto pendiente para un nuevo perfil}

\author{
Luque-de la Rosa, Antonio; Hernández-Garre, Carmen María; Fernández-Martínez, María del Mar; \\ Carrión-Martínez, Juan José \\ Docentes de audición y lenguaje itinerante: el trabajo colaborativo, reto pendiente para un nuevo perfil \\ Revista Educación, vol. 43, núm. 1, 2019 \\ Universidad de Costa Rica, Costa Rica \\ Disponible en: http://www.redalyc.org/articulo.oa?id=44057415011 \\ DOI: https://doi.org/10.15517/revedu.v43i1.28110
}

Esta obra está bajo una Licencia Creative Commons Atribución-NoComercial-SinDerivar 3.0 Internacional. 
Docentes de audición y lenguaje itinerante: el trabajo colaborativo, reto pendiente para un nuevo perfil

Itinerant hearing and language professors: collaborative work, pending challenge for a new profile

Antonio Luque-de la Rosa

Universidad de Almeria, España

aluque@ual.es

(iD http://orcid.org/0000-0001-7981-029X

Carmen Maria Hernández-Garre

Universidad de Almeria, España

cmhgarre@ual.es

(iD) http://orcid.org/0000-0002-7964-8349

Maria del Mar Fernández-Martínez

Universidad de Almería, España

mfm386@ual.es

iD http://orcid.org/0000-0001-6007-5491

Juan José Carrión-Martínez

Universidad de Almeria, España

jcarrion@ual.es

http://orcid.org/0000-0003-3115-2907
DOI: https://doi.org/10.15517/revedu.v43i1.28110

Redalyc: http://www.redalyc.org/articulo.oa?id=44057415011 
In accordance with the previous idea, coordination between hearing and language teachers and the educational center is scarce, confirming the lack of dedication to the center and/or educational communities for planning tasks, coordination, attention, etc., which hinders the coordination among these, to which it joins an apparent family disinterest and the conception as an external specialist by the educational centers. Considering this situation, it is necessary to rethink the lines of action of these teachers in order to enhance mechanisms that channel collaboration strategies, with the diverse actors involved in the educational process of the students they serve.

KEYWORDS: Hearing and language teachers, Educative counseling, Collaborative work, Educational support.

\section{INTRODUCCIÓN}

Si bien existía el perfil profesional dedicado a la atención específica de problemas relacionados con la pronunciación y el habla, el logopeda, surge al inicio de los 90 una nueva figura docente, el y la docente de audición y lenguaje, en cuya denominación se aprecia el deseo de cambio en dicho rol, al promover la superación de los planteamientos recuperadores y rehabilitadores de carácter terapéutico del logopeda para avanzar hacia una concepción de dicho profesional como potenciador del desarrollo educativo a través del lenguaje en el conjunto del alumnado, y no solamente en los niños y niñas con necesidades de apoyo educativo en este ámbito de su desarrollo.

El objeto-problema de este estudio se adentra en la en el análisis de la acción del docente de apoyo itinerante en audición y lenguaje, poniendo el foco en la cuestión del trabajo colaborativo y en la coordinación que su actuación supone en un escenario singular, en el que la dispersión del área de intervención y la propia itinerancia se proyectan sobre su actuación docente (Gobierno de España, 2011).

En este trabajo se persiguen como objetivos, de una parte, evidenciar si el modelo de intervención que llevan a cabo estos docentes especializados se sustenta en el trabajo colaborativo y la coordinación de su actuación con el conjunto de la comunidad educativa y con el equipo de orientación educativa en el que se insertan, y de otra, analizar las relaciones de colaboración que caracterizan su actuación.

Abordaremos el estado de la cuestión, la metodología y los resultados organizados estos en tres grandes categorías.

\section{MARCo TEÓRICO Y ESTADO DE LA CUESTIÓN}

Hace ya años que Santos-Guerra (1989) nos señalaba que la escuela de hoy camina hacia el paradigma de la colegialidad, participando de una concepción del proyecto educativo como desarrollo coordinado de la acción educativa. Según este autor, es un hecho constatable la incidencia y determinación de la faceta organizativa en los planteamientos didácticos que se llevan a cabo en la realidad escolar.

Dichos planteamientos organizativos habrían de incidir, entre otros aspectos, en la definición y reajuste de los roles profesionales que intervienen en dicho contexto.

$\mathrm{Si}$ hemos de tratar con problemas de cambio organizativo deberemos tomar en consideración las propiedades sistémicas de la organización y no confundir la promoción del cambio individual con el establecimiento de las modificaciones en las variables organizacionales favorables al mismo (Katz y Khan, 1983).

Ya plenamente inmersos en el siglo XXI, las teorías pedagógicas, asumidas en buena parte por prescripciones de las administraciones educativas, conforman un nuevo rol docente caracterizado por pretensiones como impulsar los planteamientos colaborativos y la coordinación en el seno de los equipos educativos.

Según Pérez-Gómez (2007), al docente contemporáneo le corresponde una tarea profesional más compleja que la mera explicación de contenidos y evaluación de rendimientos; debe diseñar, planificar, organizar, 
estimular, acompañar, evaluar y reconducir, de manera colegiada, los procesos de aprendizaje de los estudiantes en su larga trayectoria de formación como ciudadanos autónomos y responsables.

Ante el reto que supone dotar a la función educativa de un carácter colaborativo, debe tenerse en cuenta la complejidad que implica. En este sentido, uno de los riesgos que pueden interferir en la calidad de la educación y su sentido colaborativo es un exceso de burocracia administrativa que provoque la delegación de la acción educativa en las editoriales como autoras de los libros de texto (Gimeno-Sacristán, 1989), alejándose de las pretensiones que se vienen proclamando, congruentes con un modelo de profesorado autónomo, polivalente y crítico (Martínez-Bonafé, 1995). Por consiguiente, este planteamiento coordinado y colaborativo de la acción propiamente educativa debe transformar la acción docente en algo contrastable y revisable con carácter formativo.

Ante la acuciante necesidad de búsqueda de propuestas didácticas y organizativas innovadoras, el patrón de respuesta que siguen muchos profesores y profesoras no garantiza el cumplimiento de los objetivos que se demandan a los centros educativos (Echeíta-Sarrionandía, 2006). Así, la respuesta educativa a las dificultades de lenguaje se suelen organizar al margen del currículo, siendo el individualismo uno de los principales escollos de una actuación del maestro y maestra de audición y lenguaje para que la coordinación sea un eje básico (Luque de la Rosa y Carrión-Martínez, 2013, 2014).

Por contra, el modelo colaborativo persigue facilitar la interacción entre los profesores con objeto de promover el estudio, la reflexión y la planificación de la actuación educativa de forma conjunta y coordinada. En este sentido, Escudero-Muñoz (1990) nos señalaba:

La colaboración postula una acción democrática, aunque no por ello indiferenciada. Se reconoce a cada cual su perspectiva, sus conocimientos y sus habilidades, a la vez que se reclama, igualmente, una complementariedad respetuosa para indagar, explorar y fundamentar mejor la acción en curso (p. 27).

Acosta-Rodríguez (2003) y Lindsay (2003) entienden la colaboración como un proceso que debe basarse en unos principios básicos. La colaboración es una actitud, una capacidad a desarrollar hoy y mañana, se convierte en un deber de todo profesional y caracteriza al buen profesor. La capacidad colaborativa implica la cooperación con otros profesionales para posibilitar aprendizajes recibiendo uno mismo tal desempeño (Montero-Mesa, 2011).

Acosta-Rodríguez (2003) señala las siguientes características de la colaboración: “es voluntaria, está basada en la paridad, requiere establecer metas comunes, incluye una responsabilidad compartida en la toma de decisiones, implica corresponsabilidad en los resultados, está basada en compartir y poner en común recursos, es emergente" (p.149)

Según Hargreaves (2004), el individualismo, el aislamiento y el secretismo constituyen una forma particular de lo que se conoce como la cultura de la enseñanza, potenciando una falsa colaboración en el seno de las instituciones escolares, la cual deriva hacia situaciones de balcanización (selectiva, con grupos concretos, e igualmente competitiva), colaboración cómoda (superficial, sin profundizar en la planificación y carácter crítico-reflexivo) o colegialidad artificial (administrativa, obligatoria y rígida), siendo ésta la circunstancia que aparece como predominante en la realidad escolar y habiendo supuesto un escollo a la hora de implantar auténticamente dichos postulados educativos, tanto a nivel general, como en la parcela de atención a las necesidades educativas especiales (Carrión-Martínez, 2001; García-Pastor, 1995; LópezMelero, 1990; Sánchez-Palomino, 2000).

Sin embargo, a pesar de cierta profusión terminológica actual con relación a los postulados colaborativos, hay que alertar ante los intercambios superficiales o la colaboración puntual que a veces suele darse en la escuela (Imbernón-Muñoz, 1997; Parrilla-Latas, 1996), advirtiendo acerca de la necesidad de potenciar una verdadera actitud cooperativa permanente en los profesionales, para lo cual se hace preciso fomentar la creación de un clima que facilite el trabajo compartido y las metas comunes (Marcelo-García, 1995), al tiempo que potencie la interactividad, la igualdad, la definición de objetivos comunes y la mejora como finalidad 
(Goor, 1994). En este sentido, las prácticas colaborativas que se establecen entre el profesorado determinan una forma de cultura escolar y profesional (Baweja et al., 2016; Hargreaves, 2004; Pérez-Gómez, 2004.)

Dado el perfil tradicional de actuación en los centros, Castejón-Fernández y España-Ganzaráin (2004) nos señalan las siguientes cuestiones que llevan al logopeda (estos autores siguen conservando la denominación logopeda) a experimentar la necesidad de colaborar con el resto de maestros y maestras:

¿Cómo puede intervenir el logopeda sobre estas dificultades si desconoce las exigencias curriculares e interactivas del aula ordinaria y las dificultades que allí encuentra el alumno?, ¿cómo puede comprobar que la intervención sobre el lenguaje que está desarrollando resulta funcional en el contexto del aula ordinaria? (p.58)

Desde este modelo colaborativo de intervención, el y la docente de audición y lenguaje realizaría, en coordinación con los demás docentes y resto de personal especializado, una serie de funciones desde una perspectiva más amplia que la tradicional intervención clínica-directa (Galán-Galán, 2000; Valero-Matas y Silvestre-Benach, 2000). Así, los diferentes modelos de intervención desarrollados en el campo de audición y lenguaje requieren de una actuación conjunta para que sean efectivos (Atencia-Yuste, 2010).

Podría decirse que hasta ahora ha habido dos realidades que han condicionado la estructura organizativa en las escuelas. En la primera, el profesorado ha estado habituado a trabajar de manera aislada, en la segunda, la escuela ha ofrecido pocos incentivos y apoyo para una verdadera labor en equipo (Hargreaves, 2004).

No obstante, a pesar de considerar el modelo educativo-colaborativo como el más acorde al perfil del docente de audición y lenguaje, hemos de reconocer que, desde el punto organizativo y didáctico, la realidad del servicio debe regirse por el principio de flexibilidad, partiendo siempre de unos postulados de referencia curriculares-colaborativos (Luque y Carrión, 2011), pero sujetos a las matizaciones necesarias en función de múltiples factores que se dan en la realidad: itinerancias, número de niños y niñas atendidos y/o en las aulas, tipología de las necesidades de apoyo educativo, etc.

Por consiguiente, como señalan Acosta y Moreno-Santana (1999), aunque se apueste por un modelo colaborativo de intervención en el lenguaje, el maestro y/o maestra itinerante de audición y lenguaje adoptará medidas de intervención en función de las características de cada situación.

\section{Procedimientos metodológicos}

La investigación que se presenta se plantea desde un enfoque naturalista e interpretativo, pero con especial énfasis en el respecto a los datos empíricos, eludiendo la interpretación especulativa. Por la naturaleza del hecho investigado, los propios fines de la actividad investigadora, el modo de abordar el análisis e interpretación de los datos y la toma de información la metodología ha sido cualitativa. Consideramos que sólo desde esta perspectiva podemos acercarnos al conocimiento de esta realidad educativa (Goetz y LeCompte, 2010; Rodríguez-Gómez, Gil-Flores y García-Jiménez, 1996; Taylor y Bogdan, 2010) puesto que esta es cambiante y, el contexto social y los individuos que la conforman, la influyen y determinan.

Dentro de este contexto paradigmático se ha trabajado con un estudio de caso múltiple (HernándezSampieri, Fernández-Collado y Baptista-Lucio, 2015; Stake, 2006) basado en la singularidad e interés de siete contextos de actuación, los que definen cada equipo de orientación educativas en su respectivas áreas geográficas de actuación, ya que cada uno de ellos integra en su estructura de profesionales al menos un maestro o maestra itinerante de audición y lenguaje. 
TABLA 1

Tabla 1. Casos: Área geográfica/EOE $/ \mathrm{n}^{\circ}$ docentes itinerantes de audición y lenguaje

\begin{tabular}{|c|c|c|}
\hline $\begin{array}{c}\text { Denominación delárea } \\
\text { geográfica }\end{array}$ & $\begin{array}{c}\text { Código de equipo de } \\
\text { orientación educativa utilizado }\end{array}$ & $\begin{array}{c}\text { Número de maestros de } \\
\text { audición y lenguaje adscritos }\end{array}$ \\
\hline Beninar & EOEBE & 1 \\
\hline Fiñana & EOEFT & 2 \\
\hline Marmol & EOEMA & 1 \\
\hline Mónsul & EOEMO & 2 \\
\hline Murgi & EOEMU & 2 \\
\hline Sabinal & EOESA & 1 \\
\hline Urci & EOEUR & \multicolumn{2}{c|}{} \\
\hline
\end{tabular}

Fuente: Elaboración propia

Así pues, en la investigación que se presenta, los casos están representados por los equipos de orientación educativa de la provincia de Almería, España, y dentro de ellos por aquellos que tienen adscritos maestros y/o maestras de audición y lenguaje itinerantes. Con ellos se intenta analizar y comprender la actuación profesional de dichos maestros, acercándose a la práctica educativa que realizan, viviéndola y percibiéndola en su singularidad, con objeto de situar significativamente el conjunto de dimensiones que conforman esa realidad natural.

De dicho contexto de estudio, han sido seleccionados como informantes clave al total de los maestros y maestras de audición y lenguaje itinerantes, diez, adscritos a los siete equipos de orientación educativa de la citada provincia que disponen de ese perfil profesional.

Con respecto a las técnicas, la información se ha recolectado mediante entrevista abierta, observación y análisis de documentos. Las entrevistas y la observación se han desarrollado en un escenario de inmersión en los siete equipos de orientación educativa a los que están adscritos los diez maestros y maestras de audición y lenguaje itinerantes seleccionados como informantes clave, completándose con los demás informantes. Han sido entrevista abiertas, muy próximas en algunos momentos a la conversación informal, ya que el contexto de inmersión ha permitido compartir con informantes la interacción docente en situación de cotidianidad. Ello también ha permitido desarrollar la observación de forma muy natural, al igual que el acceso a los documentos en este contexto ha sido sencillo y espontáneo. No obstante, resaltamos que en todo momento el carácter investigador de la toma de información ha sido informado explícitamente y consentido.

Para hacer referencias al origen de los datos se ha codificado de la forma que se recogen en la Tabla 2. 
TABLA 2

Tabla 2: Claves (Instrumentos, Fuente y Lugar)

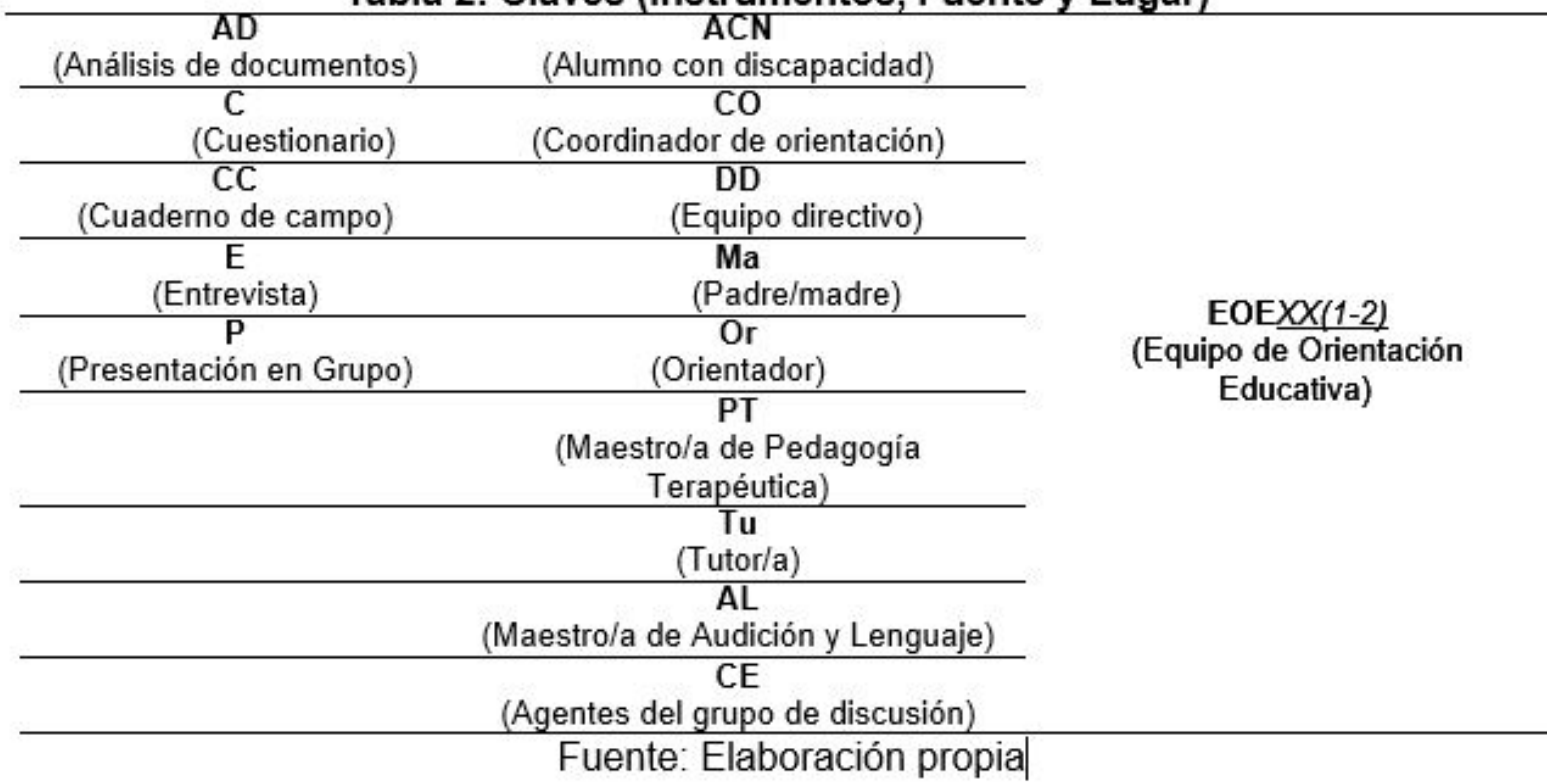

La presencia/inmersión en el escenario de alguno de los investigadores ha sido de un mes durante el cual se han desarrollado las entrevistas, se ha observado la interacción docente de los maestros de audición y lenguaje y se han cotejado diversos documentos.

En cuanto al análisis de la información recabada, tras un proceso de transcripción y depuración textual, se ha llevado a cabo un rastreo de categorías, y a partir de ahí una segmentación del texto en torno a ellas, emergiendo tres grandes categorías que estructuran el informe de resultados, colaboración con el profesorado, colaboración con la familia y colaboración con los profesionales del equipo de orientación, dentro del tema de la colaboración y coordinación como estrategia de intervención analizada en estos docentes.

\section{ANÁLISIS Y DISCUSIÓN DE RESULTADOS}

\section{Colaboración con el profesorado}

Comenzando con el proceso de colaboración con el profesorado, los maestros itinerantes de audición y lenguaje no pertenecen como miembros ni asisten a las reuniones de los órganos de coordinación educativa (equipos educativos) o de carácter colegiado (claustro) de los que forma parte el resto del profesorado del centro, debiendo aprovechar ocasiones puntuales para transmitir lo más destacable de la evolución del alumnado a los tutores, tutoras y resto de profesorado, sin llegar a poner en común de manera implicativa el conjunto de factores que conforman su desarrollo educativo (CC.AL.EOEMA1.MU2).

Si tiene algún problema o quiere darle algunas orientaciones a algún maestro no puede dárselas... O bien se las tengo que dar en otro momento o bien tengo que decir: ¡Pues mira, a éste en vez de darle hoy treinta minutos, vamos a hacer la sesión de quince porque tengo que hablar con el maestro! (E.AL.EOEBE)

En dichos encuentros se suele transmitir información generalmente administrativa (CC.AL.EOEMA2), que dista de lo que constituiría una interacción profesional docente, en la que se pongan en común criterios de actuación, se planifiquen actividades cooperativas y/o se establezcan mecanismos conjuntos:

El trabajo en equipo es importante (...). Sin embargo la logopeda no viene en esos periodos (...) así que la coordinación se reduce al comentario de los pasillos y el recreo, así como a salida/entrada del niño en el aula. (E.Tu) 
En este sentido, estos profesionales consideran necesario que el maestro de audición y lenguaje- "esté más días en los centros, como mínimo dos días” (E.AL.EOEBE), para promover momentos de trabajo colaborativo y/o coordinación con el profesorado que favorezcan la conexión con el currículo del aula ordinaria. Su falta de presencia en dichos momentos promueve su aislamiento y dificulta su integración.

Además, en opinión de determinados orientadores, dichos periodos de coordinación son necesarios para que el profesorado que manifieste interés por colaborar con esta faceta educativa no acabe renunciando a implicarse, terminando por adjudicar la total responsabilidad de los logros parciales que puedan obtenerse al esfuerzo individual del maestro y maestra de audición y lenguaje (CC.Or).

Lo bueno sería que pudieran dedicarse a orientar y asesorar el funcionamiento en las aulas pero no vemos el camino. La gente está acostumbrada a que llegue el logopeda y lo resuelva... (E.Or)

De esta manera, para parte del colectivo docente lo importante de tal figura acaba siendo que les quita los niños problema, sin implicarse en el desarrollo de ningún programa.

Es necesario que el profesorado considere que docente de audición y lenguaje - "puede ayudarle en su labor y, habría que comenzar por formar a los profesores y exigirles cierta colaboración y trabajo en equipo” - (E.Or.)

Una adecuada dedicación implicaría una organización temporal que permitiera el establecimiento en los centros de actividades innovadoras, diseño de materiales, promoción de cambios metodológicos, formación continua, etc. Igualmente, se aprecia falta de planteamientos cooperativos en la determinación y definición de los programas de intervención a desarrollar (CC.Tu):

El jefe de estudios y el maestro de PT realiza el horario del alumno con NEE al principio de curso, en colaboración con los tutores, pero la profesional de audición y lenguaje hace el suyo en función de los centros que le adjudica el EOE, por lo que no existe una coordinación como en el caso de PT. (E.PT)

La gran mayoría de los entrevistados reconoce que el diseño de sus actuaciones manifiesta una estructura paralela y desconectada del plan anual de los centros (C.AL.EOEBE, FI, MA1, MA2, MO, MU1, MU2, SA2, UR). Tan sólo uno de los maestros de audición y lenguaje manifiesta su interés en incardinar las actuaciones que desarrolla en el currículo del alumnado de aulas de educación especial (C.AL.EOESA1), pero reconoce la imposibilidad de llevar a cabo una planificación de tal labor de carácter colaborativo, conformando esta solo actividades discontinuas en función de la mayor o menor disposición del profesorado de educación especial (CC.AL.EOESA1).

No se quiere dejar de hacer referencia a los intentos que realizan algunos de estos docentes por afrontar estas limitaciones y fomentar actuaciones colaborativas y coordinadas en facetas puntuales: buscando junto con el tutor los periodos de la jornada en que las materias que se impartan en el aula sean aquéllas que muestren más dificultades para la integración (CC.AL.EOEMA2), intercambiando información sobre su actuación con el resto de profesorado, etc.

[Los tutores y yo] hablamos de los casos que compartimos aunque sea de una manera informal, espontánea, porque muchas veces, lo que es la entrevista formal, que necesita un tiempo y una revisión rigurosa de los objetivos, de los contenidos, de los avances, de los problemas que tienen los niños, de las dificultades que siguen presentando..., a veces ese análisis, digamos tan preciso y tan estructurado, casi nunca es posible hacerlo. (E.AL.EOEMO)

En estas circunstancias, la labor colaborativa entre el y la docente de audición y lenguaje itinerante y el de educación especial resulta reforzada, pudiendo verse influido por la similitud de la tarea, la proximidad de los intereses y contenidos educativos, etc.

Al tener el y la docente de educación especial poco alumnado, entre el que suele estar incluido el alumnado atendido por el y la docente de audición y lenguaje, se aseguran los momentos de intercambio de opiniones profesionales e incluso el compromiso de actuaciones conjuntas. De todas formas, dado que dicha coordinación se desarrolla en el aula durante el periodo lectivo y no se prevé su incardinación organizativa con locus horario diferenciado, se distorsiona el normal desarrollo de la dinámica en el aula y se consigue a costa de reducir la duración de determinadas sesiones de audición y lenguaje (CC.PT). 
Sin embargo, y como contraste de dicha actuación colaborativa, cabe reseñar las manifestaciones de algunos docentes de audición y lenguaje que expresan no haber participado en la elaboración de adaptaciones curriculares individuales ni ser conocedores de las programaciones que se elaboran para este tipo de alumnado.

Uno de los factores organizativos de este profesional que condiciona su coordinación con la comunidad educativa es la multidependencia funcional a la que se ve sometido al itinerar y no ser parte constitutiva a tiempo completo de un centro. Si bien debe realizar su labor diaria en diversos centros docentes a los que visita de forma periódica, su relación administrativa en los mismos se ve reducida a un control de firmas en un registro de asistencias del personal externo, manteniendo su dependencia administrativa y orgánica respecto del equipo de orientación educativa al que está adscrito.

\section{Colaboración con la familia}

En cuanto a la colaboración de su actuación con las familias, en diversas manifestaciones de los profesionales entrevistados se recoge la importancia de la adecuada colaboración familiar para impulsar la labor que el y la docente de audición y lenguaje realiza en sus sesiones semanales/quincenales (CC.AL.EOEFI), pero reconocen no poder impulsarla de forma sistemática, ya que suelen desarrollar dichos contactos de forma esporádica y a costa de suprimir alguna sesión de trabajo con los alumnos. En este sentido, si la iniciativa parte de la familia, se aprovechan los periodos de recreo o durante las sesiones (interrumpiendo parte de las mismas) y “dejando de atender al niño que tenía 'adjudicado' en esa hora” (E.AL.EOEUR).

Entonces tienes que coger en el recreo, te tienes que quedar un ratito más o ya te digo, le mermas un poquito la sesión al niño y te vas a hablar. (E.AL.EOEBE)

Otras veces se les cita para algún día venidero, pero, en cualquier caso, se suelen mantener a la espera de que la petición surja de la familia, omitiendo cualquier promoción de colaboración sistemática con esta, sin poder, por consiguiente, plantear otras actuaciones más allá de dichas atenciones puntuales a las demandas formuladas.

Esta pretensión contrasta con la asignación inicial de -"una o dos sesiones a la semana que eran para eso, para la coordinación, para entrevistas con padres, con tutores, para llevar grupos de trabajo”(E.AL.EOEMA2), la cual llega a ser inexistente en determinados casos en los que, - "al haber aumentado la demanda de logopedas en los centros y no haber aumentado el número de personal, no ha quedado tiempo para esto"- (E.AL.EOEMA2). De esta manera, se acaba quitando importancia a la promoción de dicha colaboración familiar y se potencia el absentismo inicial que los padres y madres acostumbran a manifestar por las cuestiones escolares de sus hijos.

Así, al hecho de no existir un tiempo fijado de atención a padres y madres acaba uniéndose la falta de interés de los mismos en suplir las carencias del sistema (recursos, horarios del personal...) para solventar los problemas de su hijos e hijas mediante la colaboración con el profesorado, no llegando -en la mayoría de las ocasiones-, a conocerse.

Todo esto necesitaría un tiempo adicional, que se vería incrementado de manera exponencial al considerar la dispersión geográfica y la amplitud de centros y familias asignadas (sólo considerando la faceta correctiva de orden lingüístico), encontrando además los inconvenientes propios de la disponibilidad horaria del padre y madre por motivos laborales.

\section{Colaboración con el equipo de orientación educativa}

El y la docente de audición y lenguaje ha de colaborar también con los profesionales del equipo de orientación (orientador, médico, maestro de apoyo) al que está adscrito, dedicando una sesión matinal para ello (AD9.AL.EOEMA2), aunque, según nos confirma una de las entrevistadas, existen numerosas situaciones a lo largo del curso en las que -"querrías verlos antes, preguntar algo o coordinarte en algo"(E.AL.EOEMA2), y no puedes.

En el seno de estos equipos, a pesar de la escasa periodicidad de reuniones que mantienen, se defiende la necesidad de dicha colaboración al considerar que - "a pesar de profesiones distintas y tal, quizá haya una 
conexión mayor con los profesionales que formamos los equipos que con el cuerpo docente de los colegios, los cuales hacen su tarea académica y parece que sería un trabajo más eficaz [el que realiza el y la docente de audición y lenguaje del equipo de orientación educativa] en coordinación con los orientadores."- (E.Or)

A través de dichas sesiones matinales de coordinación se ponen en común los aspectos administrativos que procedan, se diseña la actuación a desarrollar por los diversos miembros a nivel de zona, se cumplimentan los informes requeridos y se intenta adoptar un enfoque interdisciplinar en las evaluaciones e intervenciones que se desarrollan con el alumnado (CC.CO.EOEMA).

Este periodo fijo de coordinación promueve el contacto entre los diversos componentes itinerantes que atienden un mismo colegio, ya que el hecho de no disponer de suficientes espacios en los centros provoca que la distribución horaria de las visitas evite la coincidencia en los mismos.

Por otra parte, en cuanto al valor otorgado a dichas reuniones por los docentes de audición y lenguaje, existen quienes las defienden como la forma de completar la información necesaria para desarrollar su labor (CC.AL.EOEBE), frente a otros que conciben dichas reuniones como una pérdida de tiempo que les aleja de su lugar natural de trabajo, el centro educativo:

En la zona nos reunimos aquí todos los viernes como te he comentado antes. La verdad es que no tienen mucha utilidad. Bueno, algunas sí porque comentamos si a estos niños los han evaluado, cómo van, etc. Sí son útiles, pero poco. (E.AL.EOEMA1)

La forma de coordinarse a nivel provincial de los maestros itinerantes de audición y lenguaje, se constata que ha variado a lo largo de los últimos años (CC.AL.EOEBE). Al comienzo existían unas reuniones mensuales de ámbito provincial específicas para ellos en las que se pretendía poner en común líneas de actuación, se elaboraba material didáctico o informes-tipo y se formulaban propuestas de cara a la administración, mediante el establecimiento de grupos de trabajo (CC.AL.EOEFI). Dichas reuniones fueron cediendo con el tiempo al sucederse sin conseguir grandes resultados, requiriendo el desplazamiento de los profesionales de la provincia y restando tiempo a las demandas de atención o de diseño que el alumnado reclamaba (CC.AL.EOEMA1)

Tenemos reuniones a nivel de zona en Almería. Creo que están bien. No podemos ir tampoco mucho porque tenemos muchos niños. No podemos faltar tanto a los centros, pero sí, porque te enriqueces, a lo mejor unos aportan unos materiales que te vienen bien, otros te dicen su metodología y... janda, pues mira! ¡Fíjate! (E.AL.EOEMU1)

Según opiniones recogidas a lo largo del presente estudio, más bien servían para poner en común las vicisitudes acontecidas en los centros y para realizar propuestas de intenciones que el tipo/periodicidad de reunión en nada favorecía.

Esta sensación acerca de la escasa utilidad de dichas reuniones, unida a la inasistencia a las mismas de un porcentaje considerable de un colectivo poco numeroso, decantó otro modelo en el que se comenzaron a reunir por áreas geográficas (CC.AL.EOEMA1, MO).

A nivel de provincia sí ha habido algunas reuniones... Lo que pasa es que no hemos estado toda la provincia, hemos estado zona poniente/zona levante. Entonces, de forma conjunta no. Pero es que claro, donde estamos muchos pues ya se sabe (E.AL.EOESA2)

Pero la frecuencia de asistencia a dichas reuniones de área es variable sin que se llegara a establecer una estrategia de puesta en contacto o labor colaborativa entre el conjunto de los docentes de esta especialización de la provincia (CC.AL.EOEMO, SA1).

Como podemos apreciar, al perfilarse un sistema de coordinación que en nada asegura una periodicidad o productividad en las reuniones de un año para otro, se produce la ausencia progresiva de sus miembros, reforzando el aislamiento del docente itinerante de audición y lenguaje de cara al establecimiento de un rol propio, unificado y reforzado. 
Todos los años intentamos coordinarnos con alguna reunión, pero nunca se llega a seguir un mismo nivel o esquema entre todos, aunque cada uno lo hiciera a su manera. Es lo que diría yo que falta. En este equipo se está bien pero con los de otros equipos nos juntamos poco. (E.AL.EOEUR)

\section{Conclusiones}

A la vista de los resultados obtenidos, se puede considerar lo siguiente:

1) Si bien desde desde los planteamientos iniciales de los años 90 en torno al tratamiento del componente lingüístico en los centros escolares se demandaba una actuación educativa de carácter global, en donde el trabajo colaborativo y la coordinación entre docentes, resto de profesionales intervinientes en el centro escolar y familia fuese clave para el desarrollo de una nueva visión de la función del hasta entonces llamado logopeda, y ahora maestro de audición y lenguaje, el desarrollo efectivo de la dinámica docente no ha promovido y exigido dicha función, quedando pendiente el reto de un modelo más curricular y educativo en donde el trabajo colaborativo y la coordinación sea un eje esencial, lo que concuerda con dificultades para el trabajo colaborativo expuestas en distintos momentos por Hargreaves (2004), Pérez-Gómez (2007) o Santos-Guerra (1989), entre otros.

Por consiguiente, vemos que el modelo propuesto hace ya más de 25 años no guarda relación, habiendo cedido a favor de la pervivencia del establecido (clínico-logopédico), eso sí, parcialmente modificado a nivel normativo sobre la base de exigencias organizativas y curriculares (labor de equipo, concreción progresiva del currículo) que tampoco llegaron a materializarse con efectividad.

2) La coordinación se ve reducida a motivos organizativos, de colegialidad artificial, y el y la docente de audición y lenguaje no es convocado a ningún órgano de coordinación de la vida institucional de los centros en donde actúa. Se echa en falta un órgano de coordinación intercentros en el cual se pusieran en común los criterios de atención, los recursos de la zona, las demandas a la administración, etc., que consideramos importante en un modelo de intervención itinerante donde la acción docente no está integrada de forma nítida en la estructura del centro.

3) Inexistencia de espacios y tiempos para la dedicación a tareas de planificación/coordinación/atención, etc., que permitan la intervención en los centros y comunidades educativas de forma coordinada y la mejora de la eficacia de su función, compensando el desinterés familiar y proyectando una concepción alternativa a la de especialista-externo.

4) Aunque administrativamente pertenecen a un equipo de orientación educativa de carácter zonal, la multiplicidad de perfiles profesionales y la provisionalidad en dichos puestos obstaculiza cualquier dinamismo real de trabajo colaborativo y la coordinación interna del equipo.

6) Para los centros y /o alumnado que no llega a ser atendido por no existir disponibilidad horaria, no existen unos planteamientos eficaces de corte compensador al carecer de mecanismos colaborativos, asumiendo denominaciones clínicas como lista de espera.

Es por todo lo comentado, que advertimos sobre la necesidad de reformular las líneas de actuación de estos docentes con objeto de potenciar el carácter que originó su constitución, un modelo de intervención más educativo basado en el trabajo colaborativo y la coordinación docente, avanzando por tanto en el establecimiento de mecanismos que canalicen dichas estrategias de colaboración con los diversos sectores incidentes en el proceso educativo del alumnado que atienden, a este respecto el trabajo colaborativo ha mostrado su eficacia en diversas experiencias específicas de enseñanza del lenguaje como lo acreditaron Nippold, Ward-Lonergan y Fanning (2005) o muy recientemente Acosta-Rodríguez, del Valle-Hernández y Ramírez-Santana (2018).

$\mathrm{Al}$ promover la figura del maestro y maestra itinerante de audición y lenguaje con un perfil más educativo, hemos de considerar que cualquier intento de innovación asentado exclusivamente sobre los individuos, si no han tenido en cuenta las varia $\neg$ bles organizativas institucionales de los centros escolares, corre el riesgo de 
diluirse entre las dificultades, los problemas y las inercias de una organi $\neg$ zación que es generalmente resistente al cambio.

\section{REFERENCIAS}

Acosta-Rodríguez, V. M. (2003). Las prácticas educativas ante las dificultades del lenguaje. Una propuesta desde la acción. La colaboración entre logopedas, psicopedagogos, profesores y padres. Barcelona: Grupo Ars XXI de Comunicación.

Acosta-Rodríguez, V. M. y Moreno-Santana, A. M. (1999). Dificultades del lenguaje en ambientes educativos. Del retraso al trastorno específico del lenguaje. Barcelona: Masson.

Acosta-Rodríguez, V. M., Del Valle-Hernández, N. y Ramírez-Santana, G. (2018). La intervención en habilidades narrativas en alumnado con dificultades del lenguaje. Una oportunidad para el trabajo colaborativo entre logopedas, profesorado y familias. Revista de Logopedia, Foniatría y Audiología, 38 (2), 41-92. DOI: 10.1016/ j.rlfa.2018.03.001.

Atencia-Yuste, A. (2010). La intervención del maestro/a de audición y lenguaje con alumnos/as con autismo o con otras alteraciones graves de la personalidad. Revista Digital Innovación y Experiencias Educativas, 33, 1-11.

Baweja, S., Santiago, C. D., Vona, P., Pears, G., Langley, A. y Kataoka, S. (2016). Improving implementation of a schoolbased program for traumatized students: Identifying factors that promote teacher support and collaboration. School Mental Health, 8(1), 120-131.

Carrión-Martínez, J. J. (2001). Integración escolar: ¿Plataforma para la escuela inclusi-va? Málaga: Ediciones Aljibe.

Castejón-Fernández, L. A. y España-Ganzaráin, Y. (2004). La colaboración logopeda-maestro: hacia un modelo inclusivo de intervención en las dificultades del lengua $j$ je. Revista de Logopedia, Foniatría y Audiología, 24, 55-66.

Echeíta-Sarrionandía, G. (2006). Educación para la inclusión o educación sin exclusio-nes. Madrid: Narcea.

Escudero-Muñoz, J.M. (1990). El centro, como lugar de cambio educativo: la perspec $\neg$ tiva de la colaboración. En J. Gairín Sallán y S. Antúnez Marcos (Coords.), Actas del I Congreso Interuniversitario de Organización Escolar (pp. 227-286). Barcelona: UAB.

Galán-Galán, P. (2000). El logopeda como profesional del lenguaje y de la comuni-cación en los centros educativos. En J. De D. Fernández Gálvez y F. Peñafiel Martínez (Eds.), Cómo intervenir en logopedia escolar. Resolución de casos prácticos (167-183). Madrid: CCS.

García-Pastor, C. (1995). Una escuela común para niños diferentes. Barcelona: EUB.

Gimeno-Sacristán, J.(1989). Proyectos curriculares, ¿posibilidad de alcance a los profesores? Cuadernos de Pedagogía, $172,14-18$.

Gobierno de España (2011). Real Decreto 1594/2011, de 4 de noviembre, por el que se establecen las especialidades docentes del Cuerpo de Maestros que desempeñen sus funciones en las etapas de Educación Infantil y de Educación Primaria reguladas en la Ley Orgánica 2/2006, de 3 de mayo, de Educación. Boletín Oficial del Estado núm. 270, de 9 de noviembre de 2011.

Goetz, J. P. y Le Compte, M.D. (2010). Etnografía y diseño cualitativo en investigación educativa. Madrid: Morata.

Goor, M.B. (1994). Collaboration enhances education for all students. Advances in Special Education, 8, 33-51.

Hargreaves, A. (2004). Profesorado, Cultura y Postmodernidad. Madrid:Morata.

Hernández-Sampieri, R., Fernández-Collado, C. y Baptista-Lucio, P. (2015). Metodología de la investigación. México D.F.: McGraw Hill

Imbernón-Muñoz, F. (1997). La formación y el desarrollo profesional del profesorado. Hacia una nueva cultura profesional. Barcelona: Graó

Katz, D. y Kahn, R.L. (1983). Psicología social de las organizaciones. México: Trillas. 
Lindsay, G. (2003). Intervención colaborativa: logopedas y profesores trabajando juntos. En V.M. Acosta Rodríguez y A.M. Moreno Santana (Eds.), Dificultades del lenguaje, colaboración e inclusión educativa. Manual para logopedas, psicopedagogos y profesores (105-113). Barcelona: Grupo Ars XXI de Comunicación.

López-Melero, M. (1990). La integración escolar, otra cultura. Málaga: Puerta Nueva / CEC.

Luque, A. y Carrión, J.J. (2011). The teacher of hearing and language: an analysis of their itinerant model. European Journal of Investigatión in Health, Psychology and Edcucation, 1(3), 95-103.

Luque de la Rosa, A. y Carrión-Martínez J. J. (2013). Análisis del grado de satisfacción profesional del maestro de audición y lenguaje itinerante. European Journal of Edcucation and Psychology, 6(1), 55-68.

Luque de la Rosa, A. y Carrión-Martínez J. J. (2014). Análisis de la actuación docente del maestro de audición y lenguaje. Revista de logopedia, foniatría y audiología, 34(2), 68-80.

Marcelo-García, C. (1995). Formación del profesorado para el cambio educativo. Barcelona: EUB.

Martínez-Bonafé, J. (1995). El profesorado del tercer milenio. Cuadernos de Pedagogía, 240, 23-28.

Montero-Mesa L. (2011). El trabajo colaborativo del profesorado como oportunidad formativa. Participación educativa, $16,69-88$.

Nippold, M. A., Ward-Lonergan, J. M. y Fanning, J. L. (2005). Persuasive writing in children, adolescents, and adults: A study of syntactic, semantic, and pragmatic development. Language, Speech, and Hearing Services in Schools, 36(2), 125-138.

Parrilla-Latas, A. (1996). Apoyo a la escuela: un proceso de colaboración. Bilbao: Mensajero.

Pérez-Gómez, A. (2004). La cultura Escolar en la sociedad neoliberal. Madrid: Morata.

Pérez-Gómez, Á.I. (2007). Reinventar la escuela, cambiar la mirada. Cuadernos de Pedagogía, 368, 66-71.

Rodríguez-Gómez, G., Gil-Flores, J. y García-Jiménez, E. (1996). Metodología de la Investigación Cualitativa. Málaga: Ediciones Aljibe.

Sánchez-Palomino, A. (2000). Educación Especial. Respuesta a las necesidades educativas especiales desde la diversidad. Almería: Universidad de Almería.

Santos-Guerra, M.A. (julio-agosto, 1989). ¿Qué organización escolar? Cuadernos de Pedagogía, 172, 13-16.

Stake. R.E. (2006). Multiple case study analysis. New York: The Guilford Press

Taylor, S.J. y Bogdan, R. (2010). Introducción a los métodos cualitativos de investigación. Barcelona: Paidós

Valero-Matas, J. y Silvestre-Benach, N. (2000). Escuela integradora. En N. Silvestre Benach (Coord.), Sordera, comunicación y aprendizaje (pp.93-107). Barcelona: Masson.

\section{BY-NC-ND}

